\title{
Structure of the Ebola Virus Nucleocapsid Core by Single Particle Cryo-Electron Microscopy
}

\author{
Yukihiko Sugita ${ }^{1}$, Yoshihiro Kawaoka ${ }^{2,3}$, Takeshi Noda ${ }^{4}$ and Matthias Wolf ${ }^{5}$ \\ 1. Okinawa Institute of Science and Technology Graduate University, Onna, Japan. \\ 2. Institute of Medical Science,University of Tokyo, Tokyo, Japan. \\ 3. School of Veterinary Medicine, University of Wisconsin-Madison, Wisconsin, USA \\ 4. Institute for Virus Research, Kyoto University, Kyoto, Japan. \\ 5. Okinawa Institute of Science and Technology Graduate University, Onna, Japan.
}

Ebola virus (EBOV) is a deadly pathogen causing severe hemorrhagic fever in humans and non-human primates. Recent epidemics have led to thousand of deaths, chiefly in West Africa. The mortality rate of the most pathogenic strains (e.g. Zaire Ebola virus) can be as high as $90 \%$. There is no licensed vaccine or antiviral. EBOV is an enveloped virus containing a multi-layered nucleocapsid (nucleoprotein NP, VP24, VP30, VP35, RNA polymerase L) harbouring its negative-sense-RNA viral genome. The aim of this study is to determine the structure of the EBOV nucleocapsid core at high resolution using cryoelectron microscopy (cryo-EM). The gained insights will lead to better understanding of its viral assembly with detailed protein-protein and protein-RNA interactions, which will allow design of point mutants for functional studies and will serve as a structural basis for rational drug or vaccine design.

NP forms a helical structure in complex with the viral RNA genome [1,2]. This structure is the focus of the present study. VP30 and VP35 add additional layers and form a transcription- and replication-active capsid [3-5]. VP24 is involved in regulation of transcription/replication and leads to a structural change in the nucleocapsid [6,7]. So far, partial structures of these components (NP, VP24, VP30, VP35) have been reported by x-ray crystallography as well as a low-resolution structure of the nucleocapsid assembly by cryo-electron tomography [8]. Here we present the structure of the nucleocapsid-RNA complex at the intermediate resolution of $\sim 6 \AA$ using the latest instrumentation and techniques for helical processing with single particle averaging.

The C-terminal truncated mutant gene of NP (residues 1-450, NPdeltaC) was expressed in cultured mammalian cells and helical tubes were purified by $\mathrm{CsCl}$ density centrifugation. Concentrated NP in aqueous solution was rapidly frozen on holey carbon grids (Protochips, Inc) using a Vitrobot (FEI). Samples were observed at liquid nitrogen temperature on a Titan Krios TEM (FEI) at $300 \mathrm{kV}$ acceleration voltage. Images were recorded at low dose conditions (30 electrons/ $\AA^{2}$ ) using a K2 Summit direct electron detector in counting mode mounted behind an energy filter (Gatan, Inc) in zero-loss configuration. Dose fractionation and frame alignment reduced stage- or charge-induced specimen drift. Part of the dataset was recorded using a Volta phase plate (FEI). Image acquisition was automated with LEGINON [9] for defocused imaging and SERIALEM [10] for imaging with phase plate. All image processing operations were carried out with the SPRING helical particle processing software [11]. Briefly, helical tubes were manually selected in digital images, segmented, boxed and corrected for the contrast transfer function (for defocused data). Boxed segments were classified and selected by means of their power spectra. Helical symmetry parameters were determined from the best class averages using a systematic real-space search algorithm implemented in SPRING. Iterative refinement of particle alignment and symmetrized 3D-reconstruction followed "gold standard" procedure using independent datasets and alignment references. The Fourier-shell correlation $(\mathrm{FSC}=0.14)$ indicates 5.5 $\AA$ resolution. 
The three-dimensional structure of the EBOV nucleocapsid core is formed by a helical assembly of a band of tightly packed NP units. The reconstruction matches its projections and is consistent with the occasional uncoiling of the protein band observed in the phase-contrast image (Fig. 1A). Clearly resolved alpha-helical structures are located at the seam between the axial boundaries of the protein band (Fig. 1B, green). These alpha helices appear to form a zipper-like arrangement and must play a role in folding of the NP band into its quarternary biological assembly. An extended density suggests the location of the RNA strand, which is packaged with the nucleocapsid. The spacing of NP is such that each unit can interact non-specifically with a base of the viral nucleic acid. Our reconstruction suggests both a mechanism of self-assembly and for RNA-release during its disassembly [12].

References:

[1] Noda et al, PLoS Pathog 2(9) e99 (2006), 864

[2] Noda et al, J Gen Virol 91 Pt6 (2010), 1478

[3] Mühlberger et al, J Virol 73 (3) (1999), 2333-42

[4] Halfmann et al, PNAS 105 (2008), 1129

[5] Noda et al, J Infect Dis 204 Supp3 (2011), S878

[6] Noda et al, J Infect Dis 196 Supp2 (2007), S247

[7] Watanabe et al, J Infect Dis 196 Supp2 (2007), S284

[8] Bharat et al, PNAS 109(11) (2012), 4275

[9] Suloway et al, J Struct Biol 151(1) (2005), 41

[10] Mastronarde DN, J Struct Biol 152(1) (2005), 36

[11] Desfosses et al, J Struct Biol 185(1) (2014), 15

[12] The authors are grateful to R. Danev for advice on phase plate imaging and help with the setup of SerialEM. We thank OIST and the Japanese Government for generous research support.
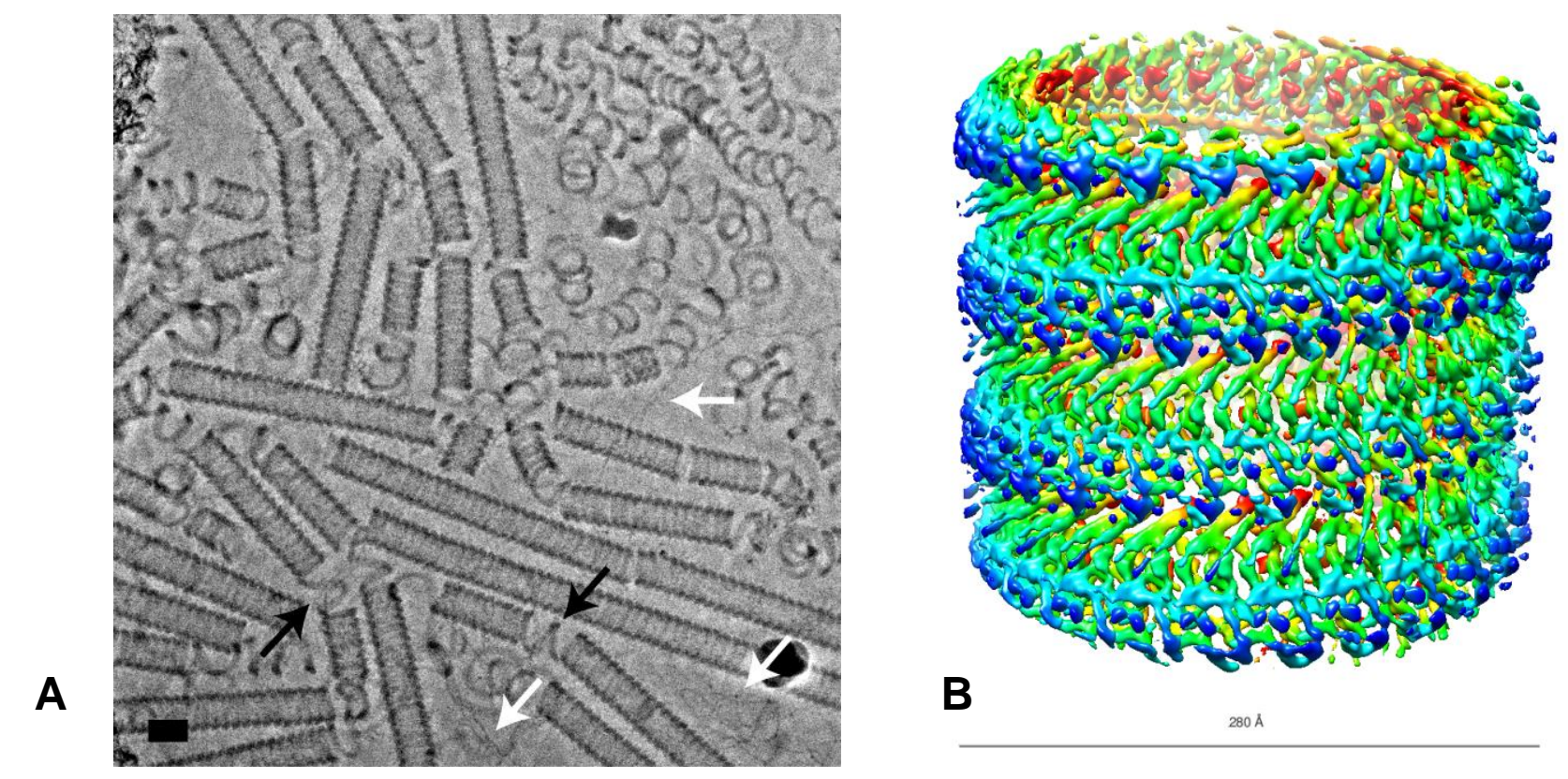

Figure 1. A. In-focus phase contrast image of EBOV NPdeltaC in amorphous ice recorded on an energy-filtered direct electron detector. Uncoiled protein bands are clearly visualized where the ordered helical tubes were kinked or broken (black arrows). White arrows indicate dissociated RNA. Scale bar $30 \mathrm{~nm}$. B. 3D reconstruction at approximately $6 \AA$ resolution from image segments. Colors depict cylinder radius (red=low < yellow < green $<$ blue=high). Scale bar $28 \mathrm{~nm}$. 